\title{
Ações educativas de enfermagem às pessoas com estoma intestinal de eliminação: revisão narrativa
}

\section{Educational nursing actions for people with intestinal elimination stoma: narrative review}

\section{Como citar este artigo:} DALMOLIN, ANCÉLICA; COMES, EDUARDO S.; COPPETTI, LARISSA C.; SIMON, BRUNA S.; SANTOS, EVELYN B.; CIRARDON-PERLINI, NARA M. O.

Ações educativas de enfermagem às pessoas com estoma intestinal de eliminação: revisão narrativa Revista Saúde (Sta. Maria). 2020; 46 (2).

\section{Autor correspondente:}

Nome: Angélica Dalmolin

E-mail: angelica_dalmolin@hotmail.com

Telefone: (55) 99962-2200

Formação Profissional: Enfermeira.

Mestre em Enfermagem pela

Universidade Federal de Santa

Maria (UFSM), Santa Maria, Rio

Grande do Sul, Brasil.

Filiação Institucional: Universidade Federal de Santa Maria

Endereço para correspondência:

Av. Roraima, $n^{\circ} 1000$. Prédio 26.

Bairro: Camobi

Cidade: Santa Maria

Estado: Rio Grande do Sul

CEP: 97 105-900

Data de Submissão:

30/03/2020

Data de aceite:

1 1/08/2020

Conflito de Interesse: Não há conflito de interesse

\section{(cc) $\mathrm{BY}-\mathrm{NC}-\mathrm{ND}$}

\author{
Angélica Dalmolin, Eduardo da Silva Gomes, \\ Larissa de Carli Coppetti, Bruna Sodré Simon, Evelyn Boeck dos Santos, \\ Nara Marilene Oliveira Girardon-Perlini
}

\section{RESUMO}

Os estomas intestinais de eliminação possibilitam desviar os efluentes fecais, por meio de um orifício na parede abdominal, o qual culmina na necessidade aderir uma bolsa coletora ao abdômen, o que pode gerar sentimentos conflituosos. As ações educativas de enfermagem, nesse contexto, visam, sobretudo, desenvolver as melhores estratégias para capacitar a pessoa com estoma para 0 autocuidado e 0 reconhecimento de possíveis complicações. 0 objetivo é identificar na literatura científica as ações de educação em saúde realizadas pela enfermagem no cuidado às pessoas com estoma intestinal de eliminação. Trata-se de uma revisão narrativa, realizada em janeiro de 2020, via Biblioteca Virtual em Saúde, a partir da estratégia: estomia OR ostomia OR estoma OR ostoma OR "estomas cirúrgicos" AND "educação em saúde". A busca inicial resultou na localização de 50 estudos, após leitura dos títulos e resumos e considerando os critérios de inclusão e exclusão 0 corpus de análise foi constituído por 13 artigos. Os resultados evidenciam a utilização de tecnologias educacionais, a conformação de grupos de apoio, as intervenções e as consultas de enfermagem como ações educativas desenvolvidas pela enfermagem, em especial no nível terciário, sendo a educação em saúde um instrumento facilitador para o cuidado, com vistas a favorecer a autonomia e independência da pessoa com estoma.

PALAVRAS-CHAVE: Estomia; Estomas Cirúrgicos; Educação em saúde; Enfermagem.

\section{ABSTRACT}

The intestinal elimination stomas make it possible to divert fecal effluents, through a hole in the abdominal wall, which culminates in the need to adhere a collecting bag to the abdomen, which can generate conflicting feelings. In this sense, educational nursing actions seek to train the person with a stoma for self-care, and it is relevant to know the scientific production related to health education actions performed by nursing in the care of this population. The objective is to identify in the scientific literature the health education actions carried out by nursing in the care of people with elimination intestinal stoma. This is a narrative review, carried out in January 2020, via the Virtual Health Library, based on the strategy: ostomy OR ostomy OR stoma OR ostoma OR "surgical stomas" AND "health education". The initial search resulted in the location of 50 studies, after reading the titles and abstracts and considering the inclusion and exclusion criteria, the corpus of analysis consisted of 13 articles. The results show the involvement of nursing in educational activities, especially at the tertiary level, with health education as a facilitating instrument for care, with a view to favoring the autonomy and independence of the person with a stoma.

KEYWORDS: Ostomy; Surgical Stomas; Health education; Nursing. 


\section{INTRODUÇÃO}

A estomia intestinal de eliminação consiste em uma intervenção cirúrgica que objetiva realizar a comunicação do intestino delgado ou grosso com o meio externo, a fim de excretar os efluentes fecais, através de uma abertura na parede abdominal. Assim, são denominadas ileostomia, quando a derivação é oriunda do intestino delgado (íleo), ou, colostomia, quando proveniente do intestino grosso (cólon), podendo ainda, ser temporárias ou definitivas".

A indicação para a confecção de um estoma está associada a algumas alterações fisiopatológicas do sistema gastrointestinal, tendo como principais etiologias as neoplasias de cólon e reto, as doenças inflamatórias intestinais, patologias congênitas e traumas ${ }^{2}$. A existência do estoma resulta em alterações no padrão habitual de vida, sendo percebida como uma experiência complexa e constrangedora, em consequência da transformação do corpo e da imagem corporal, gerando sensações de estranheza e desconforto, diante da necessidade de utilizar uma bolsa coletora de fezes aderida ao abdômen ${ }^{3}$.

A aceitação e adaptação da pessoa ao estoma é um processo individual e singular, em que as modificações vivenciadas e percebidas, necessitam de tempo para serem significadas. Nesse contexto, a assistência em saúde necessita ser diferenciada e específica, a fim de acolher as suas singularidades, sendo essencial para o processo de reabilitação físico, psicológico e social ${ }^{5}$.

Em vista disso, os cuidados de enfermagem podem transcender o corpo como objeto de cuidado, almejando promover a qualidade de vida da pessoa com estoma após a cirurgia, embasado nas premissas da integralidade e humanização ${ }^{3}$. É imprescindível que o enfermeiro seja o elo entre os conhecimentos, as informações e as orientações que subsidiarão as práticas de cuidado e do cuidar de si, sem deixar de considerar os aspectos emocionais que permeiam o processo de saúde-doença, contribuindo assim, para o retorno ao domicílio e as atividades de vida diária ${ }^{6-7}$.

O enfermeiro tem potencial para ser educador em suas atividades laborais, inserindo-se de forma ímpar no contexto da educação em saúde, comprometendo-se com o desenvolvimento da pessoa com estoma, por meio de espaços de produção, socialização e aplicação de saberes como possibilidade de qualificar a assistência prestada nos diferentes níveis de atenção a saúde ${ }^{8}$.

A educação em saúde pode preparar a pessoa com estoma e sua família para enfrentar as dificuldades advindas da confecção do estoma, através da organização e implementação de ações de cuidado direcionadas às necessidades específicas dos envolvidos. Entende-se educação em saúde como a construção compartilhada de conhecimentos, sendo balizada pela metodologia participativa, podendo auxiliar na compreensão das causas e problemas enfrentados pelos sujeitos, facilitando a adoção de estratégias para resolvê-los.

Para mais, a educação em saúde é entendida como uma relevante vertente à promoção da saúde, pois está orientada a alcançar melhorias nas condições de saúde da pessoa com estoma, proporcionando o aprofundamento do 
conhecimento científico ao cotidiano de cuidados. No contexto educacional no âmbito da saúde, os saberes singulares e diferentes se unem e convergem para a reflexão, conscientização e produção do saber coletivo .

Assim, torna-se pertinente conhecer as ações educativas desenvolvidas pela enfermagem nos diferentes níveis de atenção à saúde, a fim de compreender como ocorre o cuidado às pessoas com estoma intestinal de eliminação. Diante do exposto, tem-se como questão de revisão: "Qual a produção científica referente às ações de educação em saúde realizadas pela enfermagem no cuidado às pessoas com estoma intestinal de eliminação?". No intuito de encontrar as respostas para tal indagação, tem-se como objetivo identificar na literatura científica as ações de educação em saúde realizadas pela enfermagem no cuidado às pessoas com estoma intestinal de eliminação.

\section{MÉTODO}

Trata-se de um estudo de Revisão Narrativa da Literatura (RNL). A RNL caracteriza-se por sua abordagem ampla, permitindo a descrição de estudos e a imersão do autor sobre determinada temática, bem como o domínio acerca do objeto de estudo. Além disso, oportuniza a caracterização das produções sem exigir critérios específicos para sua realização, viabilizando a compilação das produções com a finalidade de analisar os achados e interpretá-los ${ }^{10}$.

A busca e seleção do material ocorreu em janeiro de 2020, via portal da Biblioteca Virtual em Saúde (BVS), nas seguintes bases de dados: Literatura Latino-americana e do Caribe em Ciências da Saúde (LILACS), Base de Dados em Enfermagem (BDENF), Medical Literature Analysis and Retrieval System Online (MEDLINE) e Índice Bibliográfico Espanõl de Ciências de la Salud (IBECS), a partir da estratégia: estomia OR ostomia OR estoma OR ostoma OR "estomas cirúrgicos" AND "educação em saúde". Para a seleção dos estudos não houve delimitação temporal, com vistas a proporcionar a captura do maior número possível de produções.

Definiu-se como critérios de inclusão: ser artigo; apresentar texto completo disponível em suporte eletrônico; acesso gratuito, nos idiomas português, inglês ou espanhol. Foram excluídos estudos sobre estomas respiratórios, gástricos e urinários. Os artigos indexados em mais de uma base de dados foram analisados uma única vez.

A busca inicial resultou em 50 produções. 0 processo de seleção dos artigos teve como ponto de partida a leitura criteriosa dos títulos e resumos, considerando-se os critérios de inclusão e exclusão estabelecidos, no intento de realizar o recorte temático. Assim, compuseram o corpus de análise 13 artigos, os quais foram lidos na íntegra, com vistas a extrair informações relevantes que possibilitassem responder a pergunta de revisão formulada.

Os estudos encontrados foram organizados e catalogados em um quadro sinóptico contendo as seguintes variáveis de caracterização: identificador, título, autores, periódico, ano, objetivo, delineamento metodológico e principais 
resultados, com o objetivo de reunir o conteúdo evidenciado a ser examinado. Os dados obtidos foram analisados, por meio da análise descritiva das informações, sendo estas organizadas em duas categorias, as quais foram elaboradas a partir da convergência das temáticas abordadas nos estudos que constituem o corpus dessa revisão.

\section{RESULTADOS E DISCUSSÃO}

Dos 13 artigos analisados neste estudo, seis eram estudos qualitativos ${ }^{(\mathrm{A2}, \mathrm{A6}, \mathrm{A7}, \mathrm{AB}, \mathrm{A11}, \mathrm{A12})}$, um quantitativo ${ }^{(\mathrm{A} 10)}$, dois

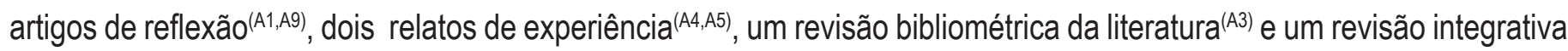
da literatura(A13). Quanto ao ano de publicação, em 2005(A3), 2006 ${ }^{(\mathrm{A} 1)}, 2010^{(\mathrm{A} 9)}, 2011^{(\mathrm{A} 8)}, 20015^{(\mathrm{A} 10)}, 2016^{(\mathrm{A} 5)}, 2018^{(\mathrm{A} 12)}$, $2019^{\left({ }^{(A 1)}\right)}$ foi publicado um estudo cada ano, dois em $2012^{(\mathrm{AG}, \mathrm{AT})}$ e três em $2017^{(\mathrm{A} 2, \mathrm{A4}, \mathrm{A13})}$. Os artigos podem ser identificados no quadro a seguir (Quadro1).

Quadro1: Identificação dos estudos que compõem a revisão de literatura.

\section{ID Referência}

Bellato R, et al. A convergência cuidado-educação-politicidade: um desafio a ser enfrentado pelos profissionais na garantia aos direitos à saúde das pessoas portadoras de estomas. Texto Contexto Enferm. 2006;15(2):334-42. Mauricio VC, et al. A visão dos enfermeiros sobre as práticas educativas direcionadas às pessoas estomizadas. Esc Anna Nery. 2017;21(4):e20170003.

Reveles AG, Takahashi RT. Educação em saúde ao ostomizado: um estudo bibliométrico. Rev Esc Enferm USP. 2007;41(2):245-50.

Rosada SR, et al. Viva bem com uma estomia: relato de experiência sobre a elaboração de uma cartilha. Rev enferm UFPE on line. 2017;11(supl. 5):2242-9.

Wild CF, et al. Educação em Saúde com estomizados e seus familiares: possibilidades para melhor qualidade de vida. Rev Enferm UFSM. 2016;6(2):290-97.

Barros EJL, et al. Gerontotecnologia educativa voltada ao idoso estomizado à luz da complexidade. Rev Gaúcha Enferm. 2012;33(2):95-101.

Martins PAF, Alvim NAT. Plano de cuidados compartilhado junto a clientes estomizados: a pedagogia freireana e suas contribuições à prática educativa da enfermagem. Texto Contexto Enferm. 2012;21(2):286-94.

Martins PAF, Alvim NAT. Perspectiva educativa do cuidado de enfermagem sobre a manutenção da estomia de eliminação. Rev Bras Enferm. 2011;64(2):322-7.

A9 Leite NSL, et al. Empowerment das famílias de crianças dependentes de tecnologia: desafios conceituais e a educação crítico-reflexiva freireana. Rev. Enferm. UERJ. 2011;19(1):152-6.

A10 Saavedra JAA, et al. Prácticas de autocuidado de pacientes enteroestomizados antes y después de intervención educativa de enfermería. Rev Enferm Inst Mex. Seguro Soc. 2015;23(2):91-8.

Carvalho DS, Silva AGI, Ferreira SRM, Braga LC. Elaboration of an educational technology for ostomized patients: peristomal skin care. Rev Bras Enferm. 2018;72(2):427-34.

Costa TC, et al. Aprender a cuidar de estoma e as contribuições de um vídeo educativo. J. nurs. health. 2018;8(3):e188301.

A13 Sousa ARA, Menezes LCG, Miranda SM, Cavalcante TB. Estratégias educativas para pessoas com estomia intestinal: revisão integrativa. Rev Enferm. Atual, 2017;81. 
Quanto a procedência das publicações, verificou-se que estas foram desenvolvidos predominantemente no Brasil, sendo quatro do Rio de Janeiro ${ }^{(A 2, A 7, A 8, A 9)}$, três do Rio Grande do Sul(A5,A6,A12), dois de São Paulo ${ }^{(A 3, A 4)}$ e um do Ceará(A13), Mato Grosso ${ }^{(A 1)}$ e o Pará(A11), respectivamente. Um dos estudos analisados era internacional, proveniente do México, especificamente da cidade de Santiago de Querétaro ${ }^{(\mathrm{A} 10)}$. Dentre os periódicos que mais publicaram sobre o tema pesquisado, destaca-se a Revista Texto e Contexto Enfermagem ${ }^{(\mathrm{A1}, \mathrm{A7})}$ ea Revista Brasileira de Enfermagem ${ }^{(\mathrm{A} 8, \mathrm{A11})}$, com dois artigos cada. Os demais periódicos apresentam uma publicação cada, sendo a Revista de enfermagem da Escola Anna Nery ${ }^{(\mathrm{A} 2)}$, Revista da Escola de Enfermagem da USP ${ }^{(\mathrm{A})}$, Revista Enfermagem UFPE on line ${ }^{(\mathrm{A} 4)}$, Revista de Enfermagem da UFSM $^{\left({ }^{(A)}\right)}$, Revista Gaúcha de Enfermagem ${ }^{(A 6)}$,Revista de Enfermagem da UERJ(A9), Revista de Enfermagem do Instituto Mexicano de Seguro Social(A10), Journal of Nursing and Health ${ }^{(\mathrm{A12})}$ e Revista Enfermagem Atual( ${ }^{(\mathrm{A} 13)}$.

No que tange a formação profissional dos autores e coautores das publicações analisadas, em sua maioria foram enfermeiros e estudantes de enfermagem ${ }^{(A 1, A 2, A 3, A 5, A 6, A 7, A 8, A 10, A 11, A 12, A 13)}$. Apenas duas produções tiveram a coautoria de uma psicóloga ${ }^{\left({ }^{A 4}\right)}$ e uma médica ${ }^{(A 9)}$. Os participantes dos estudos compreenderam adultos e idosos com estoma, família/familiares de pessoas com estoma como população alvo das ações educativas e enfermeiros, profissionais da equipe multidisciplinar, estudantes de enfermagem enquanto agentes promotores das práticas pedagógicas.

Com relação às ações educativas desenvolvidas, os autores abordaram a conformação de grupos para atividades de educação em saúde, a consulta de enfermagem enquanto prática cuidativa educacional, a elaboração de tecnologias educativas na forma de cartilhas e vídeo, com vistas a informar, orientar e capacitar a pessoa com estoma e seus familiares para o cuidado e autocuidado, a elaboração de plano de cuidado compartilhado junto ao paciente com estoma e uma intervenção educativa de enfermagem, por meio de uma apresentação no Programa Microsoft Power Point e entrega de material de apoio referente a apresentação.

Três atividades educativas foram realizadas no nível primário de atenção a saúde ${ }^{\left(\mathrm{A} 2, \mathrm{A5}^{\mathrm{A}}{ }^{12)} \text {, sendo essas em }\right.}$ uma Unidade Básica de Saúde (UBS) e na Estratégia de Saúde da Família (ESF). Duas ações de educação foram desenvolvidas no contexto de um Centro Municipal de Reabilitação ${ }^{(\mathrm{A} 2)}$ e no Serviço de Atenção à Pessoa Estomizada da Unidade de Referência Especializada em Saúde ${ }^{(\mathrm{A} 11)}$, cinco tiveram como cenário os ambulatórios de hospitais( ${ }^{(A 1, A 6, A 7, A 8, A 10)}$, correspondendo ao nível secundário e terciário de atenção à saúde. Salienta-se que três artigos analisados não apresentaram o local do estudo, pois tratavam-se de uma reflexão, uma revisão bibliométrica da literatura e uma revisão narrativa da literatura ${ }^{(\mathrm{A} 1, \mathrm{~A}, \mathrm{~A} 13)}$.

A partir da análise dos artigos emergiram duas categorias: Cuidado e educação em saúde na enfermagem: prática dialógica, política e emancipatória; e Tecnologias para o cuidado e educação: perspectivas para a construção da autonomia da pessoa com estoma. 


\section{Cuidado e educação em saúde na enfermagem: prática dialógica, política e emancipatória}

Alguns artigos analisados ${ }^{(A 1, A 7, A 9, A 5, A 13)}$ evidenciaram as ações educativas de enfermagem como prática dialógica, com dimensão política e emancipatória, voltada para a prática cidadã, a qual fortalece a autonomia e a autoestima dos sujeitos, por meio de um processo de interação e compartilhamento de saberes, experiências e vivências, entre profissional, pessoa com estoma e sua família.

A enfermagem desenvolve ações educativas no seu cotidiano laboral, pois atua na tríade da assistência, gestão e educação em saúde, perfazendo as múltiplas circunstâncias que orientam o cuidar. Sob essa ótica, as dimensões que aproximam o cuidado de enfermagem e a educação são inseparáveis, visto que os profissionais estão imbricados no processo que busca emancipar o sujeito que é cuidado, deixando este de ser dependente e tutelado pelo profissional, mas construindo de maneira contínua a sua autonomia, enquanto sujeito ativo em seu processo terapêutico ${ }^{11}$.

O cuidar e educar na prática da enfermagem é um movimento dinâmico e dialógico, possibilitado pelo envolvimento mútuo entre os participantes, na busca pela liberdade dos sujeitos, oportunizando o pensar crítico e reflexivo(A13). Para mais, o cuidado em saúde, em especial na enfermagem, tem sentido amplo, pois permeia o contexto político e social que compõe a ação de cuidar de outrem, com vistas a busca e efetivação da cidadania dos sujeitos ${ }^{12}$.

Os profissionais de enfermagem empreendem esforços para concretizar a politicidade dentro do arcabouço educativo em saúde, contribuindo para o processo emancipatório de todos os sujeitos envolvidos no cuidado, quais sejam, usuários e seus familiares, profissionais de saúde e estudantes, ampliando a politicidade como uma habilidade humana para inter-relacionar-se no contexto social e político ${ }^{(A 1)}$. Cuidado e educação são construções essencialmente políticas que repercutem na formação de sujeitos capazes de fazer sua própria história, bem como colaborar enquanto cidadão para o processo histórico da coletividade ${ }^{12}$.

Em complemento, um dos estudos analisados teve por objetivo conhecer o compartilhamento de saberes e práticas de manutenção com a estomia de pacientes atendidos em contexto ambulatorial, a partir do cuidado compartilhado(A7), o qual estava embasado pelos pressupostos do construcionismo social. Essa proposta educativa foi orientada pela compreensão de que o paciente é ativo com relação às suas demandas de cuidado e deve participar de forma efetiva na construção do plano de cuidados, associando os saberes científicos aos saberes provenientes do senso comum ${ }^{13}$.

O cuidado compartilhado desenvolvido a nível ambulatorial mediante a troca de experiências e saberes, a partir da problematização de situações relacionadas a convivência com o estoma permite 0 entrelaçar das informações e orientações entre paciente e profissional. Além disso, possibilita um momento de reflexão, em que a pessoa com estoma repensa suas práticas de cuidado, estando disponível a somar e agregar novos conhecimentos ${ }^{(A 7)}$. 
Considerando a relevância da socialização de saberes, um dos estudos analisados objetivou discutir a articulação do método freireano no trabalho em grupo junto às famílias de crianças com estoma. 0 grupo educativo abordou a perspectiva de superação das adversidades para o cuidado, contribuindo com o empoderamento das famílias a partir da educação crítica-reflexiva e assim, colaborou com o processo de construção da autonomia dos sujeitos envolvidos no cuidado ${ }^{(A 9)}$.

Oempoderamento ou empowerment, termo proveniente do inglês, constitui-se como um construto multidimensional de evolução do ser humano, resultando no desenvolver da autoconfiança, independência, controle de si e poder para decidir sobre as suas ações. Deste modo, pode auxiliar os indivíduos, grupos e comunidades a desenvolver a consciência crítica, resultando no despertar individual e/ou coletivo na busca por melhorias nas condições de saúde e de vida ${ }^{14}$.

As práticas grupais propiciam o contato de pessoas que vivenciam doenças similares, a fim de somar as experiências vividas a partir da interação grupal. Assim, essas atividades ocupam lugar de destaque no processo de aprendizagem das pessoas com estoma e seus familiares, no que tange a adaptação a nova condição de vida, contribuindo para reintegração social e para a reabilitação ${ }^{15}$.

Neste contexto do cuidado de enfermagem, um dos estudos analisados apresentou um relato de experiência sobre as ações educativas desenvolvidas com um grupo de apoio, em uma estratégia de saúde da família. A conformação do grupo permitiu que os participantes verbalizassem suas práticas de cuidado com o estoma, a higienização e a troca do equipamento coletor, a partir do senso comum, pautado nas experiências singulares de cada pessoa, sanando as dúvidas existentes(A5).

Além disso, foram abordados aspectos relacionados a alimentação, ao hábito intestinal, ao retorno às atividades de vida diárias e a sexualidade, no intento de minimizar as repercussões advindas com a confecção do estoma ${ }^{(A 5)}$. Assim, evidencia-se que as ações educativas de enfermagem apresentam potencialidades emancipadoras, sendo uma construção política que possibilita ampliar a cidadania da pessoa com estoma, a partir de práticas dialógicas que refletem de forma positiva na qualidade de vida.

\section{Tecnologias para o cuidado e educação: perspectivas para a construção da autonomia da pessoa com estoma}

As ações educativas de enfermagem convergem para o desenvolvimento do ensino e aprendizagem para o cuidado e autocuidado, pois são possibilidades de produção e aplicação dos saberes científicos na prática clínica, nos diferentes cenários de atenção à saúde. Assim, buscam superar as dificuldades associadas ao processo de saúdedoença, a fim de oferecer perspectivas para o desenvolvimento da autonomia, favorecendo a adaptação à nova realidade de vida, respeitando as singularidades de cada pessoa. 
Nessa perspectiva, alguns artigos analisados desenvolveram tecnologias para o cuidado e educação, do tipo imprensa, apresentadas na forma de cartilhas ${ }^{(\mathrm{A4}, \mathrm{AG})}$ e guia de orientação( ${ }^{(\mathrm{A} 11)}$, com vistas a facilitar a compreensão da pessoa com estoma e de seus familiares sobre o manejo do estoma, o cuidado com a pele periestomal, a higienização e a troca da bolsa coletora. O material educativo elaborado nestes estudos, inicialmente, contém uma parte introdutória que discorre acerca da definição do que é um estoma e pele periestomal, bem como as características dos efluentes drenados, os diferentes equipamentos de coleta para as excreções, os tipos de dermatites periestoma e os cuidado necessários $^{(\mathrm{A4}, \mathrm{A6}, \mathrm{A11})}$.

As tecnologias educacionais desenvolvidas na forma de cartilha foram validadas junto ao público-alvo e a profissionais especialistas na temática, a fim de reconhecer suas possibilidades terapêuticas e educativas como recurso para a construção do conhecimento sobre o processo de saúde-doença e ensino-aprendizagem ${ }^{(A 4, A 6)}$. O guia de orientações foi confeccionado a partir das experiências e vivências de pacientes com estoma, por meio da realização de quatro encontros de grupo focal, propiciando a interação entre os participantes e o desenvolvimento de uma intervenção educativa sobre os cuidados com o estoma e pele periestomal, sendo necessário, ainda, realizar sua validação(A11).

A elaboração deste tipo de material possibilita uma abordagem diferenciada, pois reúne recursos textuais simples, por meio de frases curtas, com uma linguagem acessível e de fácil compreensão, associadas a ilustrações cuidadosamente selecionadas, as quais enfatizam aspectos importantes para o cuidado, instigando o interesse do leitor ${ }^{16}$. A utilização de tecnologias na prática da enfermagem decodifica o conhecimento científico de maneira clara e objetiva, sendo um recurso pedagógico que possibilita a integração dialógica entre profissional e pessoa com estoma, favorecendo a adaptação e reabilitação frente a nova condição de vida.

Considerando a relevância das ações educativas de enfermagem, um dos estudos analisados teve por objetivo determinar o nível de conhecimento sobre as práticas de autocuidado em pacientes com estoma, antes e depois de uma intervenção educativa de enfermagem realizada no contexto hospitalar. A intervenção foi desenvolvida a partir uma apresentação em Power point, abordando aspectos referente as práticas de autocuidado e necessidades de alimentação, por meio de um enfoque interativo, estimulando as pessoas com estoma a expressar suas dificuldades, dúvidas e anseios. Ao final da atividade foi entregue a cada participante um material de apoio referente a apresentação( ${ }^{(A 10)}$.

Os resultados do estudo revelam que o nível de conhecimento sobre as práticas de autocuidado aumentou após a intervenção educativa de enfermagem, sendo estes recursos indispensáveis, pois podem ser utilizadas pelo enfermeiro no seu cotidiano de trabalho, com vistas a aderência do público-alvo as atividades de autocuidado. Além disso, são uma possibilidade de aplicar de forma imediata os conhecimentos adquiridos e resolver os problemas vividos diante da presença do estoma(A10). 
Outro estudo analisado objetivou conhecer a percepção de pessoas com estoma e de seus familiares acerca da possibilidade de utilizar um vídeo educativo como recurso estratégico para educação em saúde. Os resultados evidenciam a tecnologia educativa como um recurso eficiente para educação em saúde dessas pessoas, pois oferece subsídios que facilitam e fortalecem as aptidões para o autocuidado, potencializando o desenvolver da autonomia e refletindo positivamente na qualidade de vida ${ }^{\left({ }^{(12)}\right)}$.

A enfermagem contribui com a autoafirmação das pessoas com estoma, por meio da realização de ações pedagógicas que visam empoderá-las para alcançar a autonomia e independência ${ }^{17}$. As metodologias educativas tornam-se instrumentos adequados para o cuidado, pois são guiadas por uma prática dialógica que culmina na reflexão e, como consequência, tem-se a ação dos sujeitos na busca pelo autocuidado, a partir da consciência de suas reais necessidades ${ }^{(A 8)}$.

A utilização dessas estratégias de ensino precisa atender as individualidades das pessoas, considerando 0 contexto socioeconômico e cultural em que estão inseridos, para assim reconhecer os fatores que podem facilitar 0 desenvolvimento das habilidades para 0 cuidado e cuidar-se ${ }^{18}$. A inserção do enfermeiro como protagonista das intervenções educativas permite repensar a prática da enfermagem com relação o cuidado da pessoa com estoma e de sua família ${ }^{3}$.

Contudo, há de se pensar que as pessoas com estoma necessitam de cuidados para além dos aspectos fisiológicos e de manuseio do estoma e da bolsa coletora, almejando a construção de um cuidado integral, o qual considera os aspectos psicossociais envolvidos nas mudanças advindas com a estomização. Nesse contexto, um dos estudos salienta a importância de que as orientações de enfermagem rompam os paradigmas biomédicos, com vistas a considerar o indivíduo como um todo, a partir do meio sociocultural em que está inserido, uma vez que esses fatores repercutem diretamente na reabilitação dessas pessoas ${ }^{(\mathrm{A} 2)}$.

O enfermeiro ao considerar as mudanças vividas no cotidiano da pessoa com estoma, no que tange as suas atividades sociais e laborais, passará a gerir o cuidado de maneira mais ampla, considerando os múltiplos fatores intervenientes que culminam no desajuste emocional e psicológico do paciente com estoma e que, muitas vezes, levam ao isolamento social. Assim, poderá contribuir com o processo de aceitação e adaptação a nova conformação do corpo, por meio de estratégias que estimulem a pessoa a buscar dentro de si aspectos que auxiliem a re(significar) essa nova fase da vida, valorizando pontos positivos que possam facilitar e colaborar com seu bem-estar ${ }^{19}$. Contudo, um dos estudos analisados revela a necessidade de melhorar o preparo/formação dos profissionais de enfermagem para assistir essas, considerando a complexidade que envolve o viver e conviver com um estoma ${ }^{(A 3)}$. 
Diante do exposto, as ações educativas de enfermagem implicam na construção coletiva do saber, resultando em possibilidades de transformar a realidade vivida, de modo que a pessoa com estoma seja ativa no seu processo de aprendizagem para o autocuidado. Assim, a prática pedagógica do cuidado não pode ser fragmentada, pois precisa ter uma visão holística e integral do indivíduo, a fim de promover a saúde e desenvolver as habilidades necessárias para resgatar a autonomia e confiança da pessoa com estoma, facilitando sua reabilitação.

\section{CONSIDERAÇÕES FINAIS}

A partir do desenvolvimento deste estudo foi possível vislumbrar que a enfermagem pensa, organiza e desenvolve ações de educação em saúde que propiciam o envolvimento da pessoa com estoma e da família no seu processo terapêutico, como uma maneira de compartilhar o cuidado. A utilização de tecnologias educacionais, a conformação de grupos de apoio, as intervenções e as consultas de enfermagem são estratégias implementadas pelos profissionais, possibilitando a construção de um saber coletivo e cooperativo, que valoriza o conhecimento advindo do senso comum.

Desse modo, as evidências indicam a educação em saúde como um instrumento de prática social, em que 0 cuidar na enfermagem também pode ser percebido como um ato político, efetivando os direitos à saúde e a construção da cidadania dessas pessoas. Dentre os locais em que as atividades foram desenvolvidas, destaca-se o nível terciário de atenção à saúde como principal cenário das práticas educativas. Com isso, identifica-se uma lacuna na perspectiva do cuidado educacional, no que tange ao nível primário, sendo revelada pela incipiência dos estudos analisados.

O nível primário de atenção a saúde constitui-se como uma via para a continuidade do cuidado à pessoa com estoma quando esta retorna ao seu domicílio. Assim, a fragmentação das práticas educativas no contra referência para 0 serviço básico de saúde impossibilita a efetivação das redes de atenção, podendo ser fator interveniente para o processo de reabilitação da pessoa com estoma.

Espera-se que essa revisão possa estimular os profissionais de enfermagem, em especial, os que atuam no nível primário a planejar e implementar ações de educação em saúde, bem como programas de cuidado ao paciente com estoma e sua família, com vistas a subsidiar o desenvolvimento de habilidades para o autocuidado e cuidado face a nova condição de vida. Como limitações do estudo indicamos a restrição do corpus, o que restringe as evidências analisadas e impossibilita generalizações. 


\section{REFERÊNCIAS}

1. Rocha JJR. Estomas intestinais (ileostomias e colostomias) e anastomoses intestinais. Medicina (Ribeirão Preto) [Internet]. 2011;44(1):51-6. DOI: 10.11606/issn.2176-7262.v44i1p51-56

2. Almeida EJ, Silva AL. Caracterização do perfil epidemiológico dos estomizados em hospitais da Secretaria do Estado de Saúde do Distrito Federal. ESTIMA. 2015;3(1):11-6.

3. Marques ADB, Amorim RF, Landim FLP, Moreira TMM, Branco JGO, Morais PB, et al. Body consciousness of people with intestinal stomach: A phenomenological study. Rev Bras Enferm [Internet]. 2018;71(2):391-7. DOI: http://dx.doi.org/10.1590/0034-7167-2016-0666.

4. Lenza NFB, Buetto LS, Vieira FS, Oliveira MS, Teles AAS, Sonobe HM. Necessidades do estomizado intestinal em seguimento oncológico: revisão integrativa. Rev enferm UFPE on line. 2015; 9(6):8715-24.

5. Sousa ARA, Menezes LCG, Miranda SM, Cavalcante TB.2017. Estratégias educativas para pessoas com estomia intestinal: revisão integrativa. Rev. Enferm. Atual In Derme. 2017;81(19).

6. Gomes B, Martins SS. A Pessoa Estomizada: análise das práticas educativas de enfermagem. ESTIMA. 2016; 14(3):146-53.

7. Freire DA, Angelim RCM, Souza NR, Brandão BMGM, KMS Torres, Serrano SQ. Autoimagem e autocuidado na vivência de paciente estomizados: o olhar da enfermagem. Rev Min Enferm. 2017;21:e-1019.

8. Maurício VC, Souza NVDO, Costa CCP, Dias MO. A visão dos enfermeiros sobre as práticas educativas direcionadas as pessoas estomizadas. Esc Anna Nery. 2017;21(4):e20170003. DOI: 10.1590/2177-9465-EAN-20170003.

9. Silva LD, Beck CLC, Dissen MC, Tavares JP, Budó MLD, Silva HS. O enfermeiro e a educação em saúde: um estudo bibliográfico. Revista de Enfermagem da UFSM. 2012; 2(2):412-419. 
10. Brum CN, Zuge SS, Rangel RF, Freitas HMB, Pieszak GM. Metodologias da pesquisa para a enfermagem e saúde. $1^{\text {a }}$ edição. Porto Alegre: Moriá, 2015.

11. Freire P. Pedagogia da autonomia: saberes necessários à prática educativa. $58^{a}$ edição. São Paulo: Paz \& Terra, 1997.

12. Demo P. Pesquisa: princípio científico e educativo. 12ª edição. São Paulo: Cortez, 2006.

13. Teixeira MLO, Ferreira MA. Cuidado compartilhado: uma perspectiva de cuidar do idoso fundamentada na educação em saúde. Texto Contexto Enferm. 2009; 18(4): 750-8.

14. Peterson NA. Empowerment Theory: Clarifying the Nature of HigheriOrder Multidimensional Constructs. Am J Community Psychol. 2014; 53(96):96-108.

15. Dias SM, Gonçalves FG. Percepções de familiares com criança estomizada sobre grupo de apoio. ESTIMA. 2015; 13(2).

16. Teles LMR, Oliveira AS, Campos FC, Lima TM, Costa CC, Gomes LFS et al. Construção e validação de manual educativo para acompanhantes durante a o trabalho de parto e parto. Rev Esc de Enferm da USP. 2014; 48(6): 977-84.

17. Barba PD, Bittencourt VLL, Kolankiewicz ACB, Loro MM. Demandas de cuidado de pacientes oncológicos estomizados assistidos na atenção primária à saúde. Rev enferm UFPE on line. 2017; 11(8):3122-3129.

18. Silva J, Sonobe HM, Buetto LS, Santos MG, Lima MS, Sasaki VDM. Estratégias de ensino para o autocuidado de estomizados intestinais. Rev Rene. 2014; 15(1):166-73.

19. Vasconcellos FM, Xavier ZDM. O enfermeiro na assistência do cliente colostomizado baseado na teoria de Orem. Revista Recien. 2015; 5(14):25-37. 